\title{
Space time and the passage of time
}

\author{
George F R Ellis, University of Cape Town
}

August 28, 2012

\begin{abstract}
This paper examines the various arguments that have been put forward suggesting either that time does not exist, or that it exists but it's flow is not real. I argue that (i) time both exists and flows; (ii) an Evolving Block Universe ('EBU') model of spacetime adequately captures this feature, emphasizing the key differences between the past, present, and future; (iii) the associated surfaces of constant time are uniquely geometrically and physically determined in any realistic spacetime model based in General Relativity Theory; (iv) such a model is needed in order to capture the essential aspects of what is happening in circumstances where initial data does not uniquely determine the evolution of spacetime structure because quantum uncertainty plays a key role in that development. Assuming that the functioning of the mind is based in the physical brain, evidence from the way that the mind apprehends the flow of time prefers this evolving time model over those where there is no flow of time.
\end{abstract}

\section{Space time and the Block Universe}

In this section I briefly summarize the usual representation in relativity theory of space-time as an unchanging block universe, and the associated view that the change of time is an illusion.

The nature of spacetime in both special and general relativity has lead some to a view that the passage of time is an illusion [72, 12, 39, 40. Given data at an arbitrary time, it is claimed that everything occurring at any later or earlier time can be uniquely determined from that data, evolved according to deterministic local physical laws (this is formalized in standard existence and uniqueness theorems [49]). Consequently, nothing can be special about any particular moment; there is no special "now" which can be called the present. Past, present and future are equal to each other, for there is no surface which can uniquely be called the present.

Such a view can be formalized in the idea of a Block Universe [59, 70, 17]: space and time are represented as merged into an unchanging spacetime entity, with no particular space sections identified as the present and no evolution of spacetime taking place. The universe just is: a fixed spacetime block, representing all events that have happened and that will happen. This representation implicitly embodies the idea that time is an illusion: time does not "roll on" in this picture. All past and future times are equally present, and the present "now" is just one of an infinite number. Price [67] and Barbour [7] in particular advocate such a position. Underlying this, as emphasized by Barbour, is the idea that time-reversible Hamiltonian dynamics provides the foundation for physical theory in general and gravitation in particular. Occasionally cosmology or astrophysics takes into account time-irreversible physics, for example nucleosynthesis in the early universe or the late phases of gravitational collapse, but the notion of the present as a special time remains absent.

The problem with this view is that it is profound contradiction with our experiences in everyday life, and in particular with the way science is carried out. Scientific theories are developed and then tested by 
an ongoing process that rolls out in time: initially the theory does not exist; it is developed, tested, refined, finally perhaps accepted: is it really plausible that all of this process is an illusion, as some claim? Can it really be that "Time is real but flow is not" (Davies [17]), or "Time does not exist" (Barbour [7, Rovelli 69])? If time is an illusion, how can the mind generate this illusion, when (assuming the validity of present day neuroscience) the mind is based in the brain - a physical entity, governed by the laws of physics?

By contrast to this view, Broad already in 1923 [11] argued that the true nature of spacetime is best represented as an Emergent Block Universe (EBU), a spacetime which grows and incorporates ever more events, "concretizing" as time evolves along each world line [24]. Unlike the standard block universe, it adequately represents the differences between the past, present, and future, and depicts the change from the potentialities of the future to the determinate nature of the past. This is the view I present in this chapter - the claim "time is an illusion" results from using an inadequate model of spacetime.

\section{Time and the Emerging Block Universe}

In this section I summarize the alternative representation of space-time as an ever-growing emergent block universe, embodying the view that the ongoing flow of time is a key physical aspect of reality; and relate this to the classical physics concept of the nature of time.

How do we envisage spacetime and the objects in it as time unrolls? To motivate the EBU model of reality, consider the following scenario [24]: A massive object has rocket engines attached at each end that allow it to move either left or right. The engines are fired alternately by a computer, which produces firing intervals and burn times based on a sensor activated by the random decays of a radioactive element [56]. These signals select the actual spacetime path of the object from the set of all possible paths. Due to the quantum uncertainty inherent in radioactive decay 38, 1, 44, the realized path is not determined by the initial data at any previous time: which potential path becomes actual cannot be predicted, it is determined as it happens (see Figure 1).

Because the objects are massive and hence produce spacetime curvature, spacetime structure itself is undetermined until the object's motion is determined by the specific radioactive decay that takes place. Instant by instant, spacetime structure changes from indeterminate to definite. Thus a definite spacetime structure comes into being as time evolves. The random element introduced through the irreducible uncertainty of quantum events ensures that there is no way the future spacetime can be predicted from the past: what will actually happen is not determined until it happens. Second by second, one specific evolutionary history out of all possibilities is chosen, takes place, and becomes cast in stone (sometimes literally). The future is uncertain and indeterminate until local determinations have taken place at the spacetime event "here and now," designating the present on a world line at a specific instant; thereafter this event is in the past, having become fixed and immutable, with a continually new event on the world line designating the present.

The EBU model of spacetime represents this situation(see Figure 2): time progresses, events take place, and history is shaped. This is represented through a growing spacetime diagram, in which the past is represented as a usual block universe, but now existing only from the start of space time up to the everchanging surface representing the present. Even the nature of future spacetime, along with the physical events that occur in it, is uncertain; unlike the past, the future does not yet exist, it is just a potentiality; hence it is not represented in the diagram as part of the presently existing spacetime. The passing of time marks the change from indefinite (not yet existing) to definite (having come into being); the present marks the instant at which we can act and change reality. Space time grows as time inexorably evolves: at each 


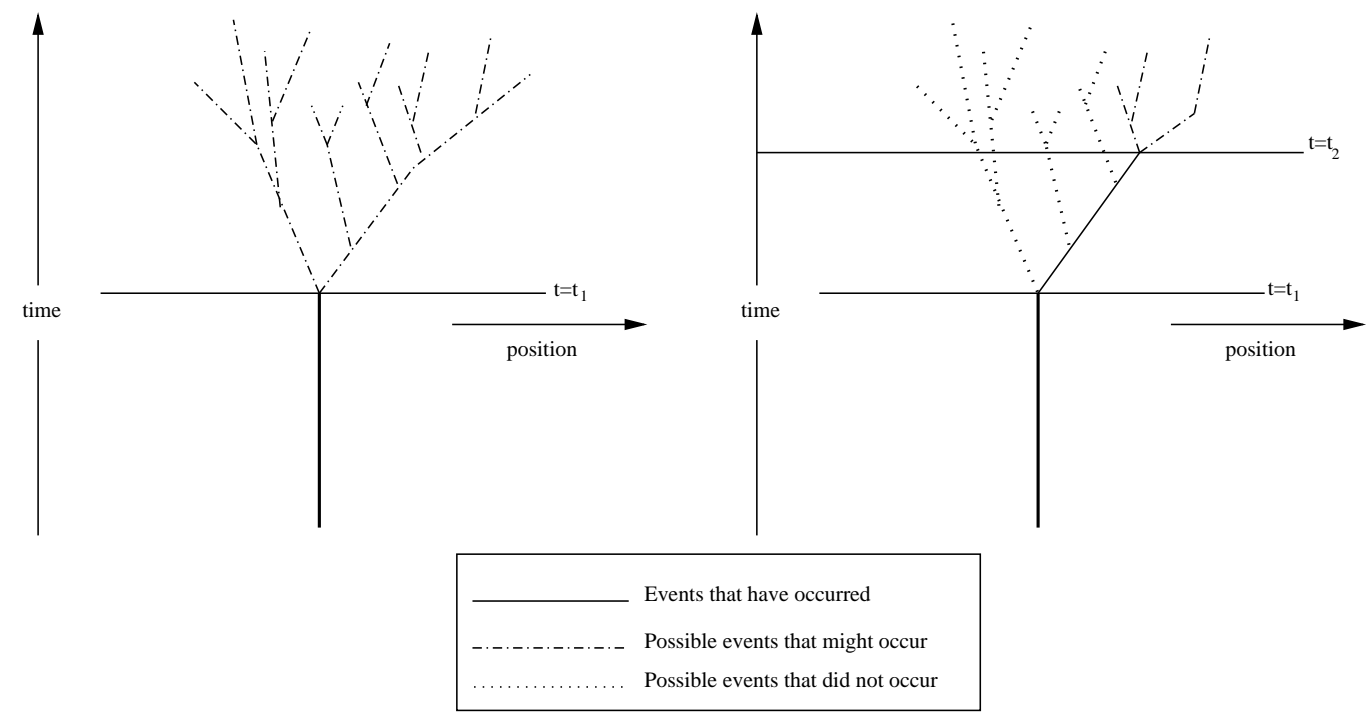

Figure 1: Motion of a particle world line controlled in a random way, so that what happens is determined as it happens. On the left events are determined till time $t_{1}$ but not thereafter; on the right, events are determined till time $t_{2}>t_{1}$, but not thereafter. Spacetime is unknown and unpredictable even in principle before it is determined (because that choice is based on the randomness of quantum decay of radioactive particles). The time at which it is determined inexorably moves on, and given this physical context, this unfolding cannot be stopped, changed, or reversed.

new instant every previous present has become part of the past [24.

The proposed view is thus that spacetime is continually extending to the future as events develop along each world line in a way determined by the complex of causal interactions; these shape the future, including the very structure of spacetime itself. The EBU continues evolving along every world line until it reaches its final state, resulting in an unchanging Final Block Universe at the end of time. One might say that then time has changed into eternity. It is this Final Block Universe that is usually represented in spacetime diagrams; but it only exists when time has everywhere run its course.

\subsection{The paradox}

This model of spacetime is obviously far more in accord with our daily experience than the standard Block Universe picture; indeed everyday data, including the apparent passage of time involved in carrying out every single physics experiment, would seem to decisively choose the EBU over the Block Universe. The evidence seems abundantly clear. Why then do some physicists prefer the latter? If the scientific method is to abandon a theory when the evidence is against it, why do some hold to it?

This counter viewpoint is put succinctly by Sean Caroll in a blog: 1

"The past and future are equally real. This isn't completely accepted, but it should be. Intuitively we think that the 'now' is real, while the past is fixed and in the books, and the future hasn't yet occurred. But physics teaches us something remarkable: every event in the past and future is

\footnotetext{
${ }^{1}$ http://blogs.discovermagazine.com/cosmicvariance/2011/09/01/ten-things-everyone-should-know-about-time/
} 

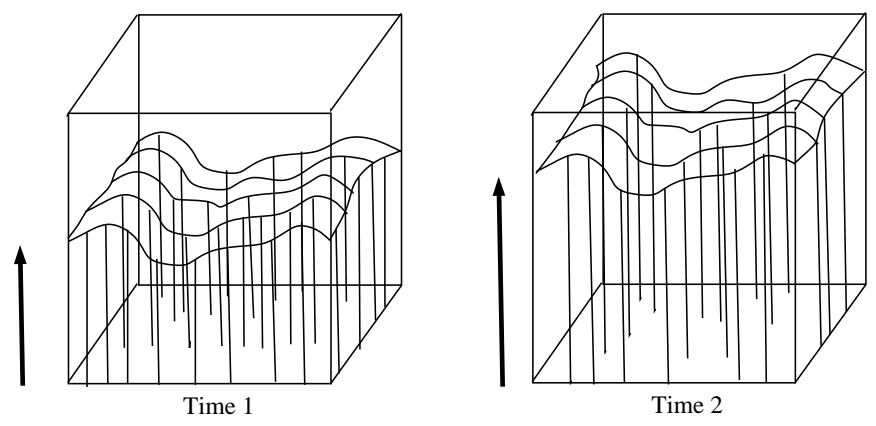

Figure 2: An evolving curved space-time picture that takes the flow of time seriously. Time evolves along each world line, extending the determinate spacetime as it does so. You cannot locally predict uniquely to the future from data on any constant 'time' surface, because of quantum uncertainty. This is true both for physics, and for the spacetime itself: the developing nature of spacetime is determined by the evolution of the matter in it. A key example is the process whereby quantum fluctuations determine seed spacetime inhomogeneities during the inflationary era in the early universe.

implicit in the current moment. This is hard to see in our everyday lives, since we're nowhere close to knowing everything about the universe at any moment, nor will we ever be - but the equations don't lie. As Einstein put it, 'It appears therefore more natural to think of physical reality as a four dimensional existence, instead of, as hitherto, the evolution of a three dimensional existence." "

But the question is which equations, and when are they applicable? As emphasized so well by Eddington ([19]:246-260), our mathematical equations representing the behaviour of macro objects are highly abstracted versions of reality, leaving almost all the complexities out. The case made in [26] is that when true complexity is taken into account, the unitary equations leading to the view that time is an illusion are generically not applicable except to isolated micro components of the whole. The viewpoint expressed by Carroll supposes a determinism of the future that is not realised in practice: inter alia, he is denying the existence of quantum uncertainty in the universe we experience. But physics experiments show that uncertainty to be a wellestablished aspect of the universe [38, 44, and it can have macroscopic consequences in the real world, as is demonstrated by the historic process of structure formation resulting from quantum fluctuations during the inflationary era [18. These inhomogeneities were not determined until the relevant quantum fluctuations had occurred, and then become crystalized in classical fluctuations; and they were unpredictable, even in principle.

Actually, the EBU proposal does not contradict the first part of the Einstein quote given in Carroll's blog. The core issue not touched on in that quote is where lies the future boundary of the 4-dimensional spacetime advocated by Einstein. In the usual Block Universe picture, it is taken to be at the end of time. In the EBU, it corresponds to the ever-changing present time.

The prime issue arising is that the spacetime view of special relativity denies the existence of any preferred time slices, whereas the claimed existence of the present in the EBU is certainly a preferred time surface (at each instant, it is the future boundary of the 4-dimensional spacetime). I will deal with this objection in the following section, after first looking at common physics views of the passage of time in the rest of this section. An array of further arguments for the claim "time is an illusion" have been made by philosophers and physicists: they are conveniently summarized in the Spring, 2012 special issue of Scientific American 
72]2. I will turn to them in 9 I then consider the way the Block Universe view relates to theories of the mind ( (6): a key problem for that view. Next I consider how the EBU picture may be altered when one takes quantum issues into account (\$2.3), and point out how it relates to the arrow of time issue (\$8.1) and solves the chronology protection question ( 8 8.2). Finally I reflect on the nature of time in relation to the EBU proposal (\$9).

\subsection{The classical physics of the passage of time}

There are no problems with the existence or passage of time in standard physics textbooks on classical mechanics, see for example The Feynman Lectures in Physics [36].

\subsubsection{Reversible Dynamics}

A standard example is a Simple Harmonic Oscillator ('SHO') with equation of motion

$$
F=m \frac{d^{2} q}{d t^{2}}=-k q
$$

and solution

$$
q(t)=A \cos (\omega t-\phi), p(t)=m \frac{d q}{d t}=-m A \omega \sin (\omega t-\phi)
$$

where $\omega:=\sqrt{\frac{k}{m}}$. As time evolves, the oscillator oscillates, with its state at time $t$ given by $\{q(t), p(t)\}$. Standard texts discussing the SHO do not question the existence or flow of time.

More generally for dynamical systems with $N$ variables $x_{i}$ :

$$
d x_{i} / d t=f\left(x_{j}\right)(i, j=1-N)
$$

the solution $x_{i}(t)$ represents how the variables change as time flows steadily on 3 In these cases knowledge of the state at any time $t_{0}$ enables deduction of the state at all earlier and later times; the system is timereversible and predictable, and evolves with time according to this equation (indeed the very purpose of these equations is to predict this time evolution). A particular case is Hamiltonian dynamics where

$$
\begin{aligned}
d p_{i} / d t & =-\frac{\partial}{\partial q_{i}} \mathcal{H}\left(q_{j}(t), p_{j}(t)\right), \\
d q_{i} / d t & =+\frac{\partial}{\partial p_{i}} \mathcal{H}\left(q_{j}(t), p_{j}(t)\right),
\end{aligned}
$$

$(i, j=1-N)$. There may be constraints, perhaps involving first and second spatial derivatives:

$$
C_{m}\left(p_{i}, q_{j}, q_{j, k}, q_{j, k l}\right)=0
$$

$(m=1-M)$ where $q_{j, k}:=\partial q_{j} / \partial d x^{k}$. Then these must be preserved under the time evolution:

$$
\{(4),(5)\} \Rightarrow d C_{m} / d t=0 .
$$

\footnotetext{
${ }^{2}$ see also [51, 50, 2 for recent reviews of these issues, with references.

${ }^{3}$ The SHO is of this form if $x_{i}=x, x_{2}=p$.
} 
Then provided the constraints are initially satisfied at time $t_{0}$, the past and the future are uniquely determined for some time interval $\left[T_{-}, T_{+}\right]$containing $t_{0}$ :

$$
\left\{\left(p_{i}\left(t_{0}\right), q_{i}\left(t_{0}\right)\right): C_{m}\left(t_{0}\right)=0\right\} \Rightarrow\left\{\left(p_{i}(t), q_{i}(t)\right): T_{-}<t<T_{+}\right\} .
$$

The time development of the system is given by these equations. Three comments:

Explicit time dependence: The case $\mathcal{H}\left(q_{j}(t), p_{j}(t), t\right)$ where $\partial \mathcal{H} / \partial t \neq 0$ breaks time translation invariance and explicitly invokes preferred times in the dynamics. I exclude this case in what follows.

Limited prediction times: generically one or both of $T_{-}, T_{+}$will be finite 49 . Except for comments on the chronology protection question ( $(\sqrt{8.2})$, I will not consider such global issues here.

First integrals: for any function $f\left(q_{i}, p_{i}, t\right)$ equations (4), (5) imply the time derivative

$$
\begin{aligned}
d f\left(q_{i}, p_{i}, t\right) / d t & =\left(\partial f / \partial q_{i}\right)\left(d q_{i} / d t\right)+\left(\partial f / \partial p_{i}\right)\left(d p_{i} / d t\right)+\partial f / \partial d t \\
& =\left(\frac{\partial f}{\partial q_{i}} \frac{\partial \mathcal{H}}{\partial p_{i}}-\frac{\partial \mathcal{H}}{\partial q_{i}} \frac{\partial f}{\partial p_{i}}\right)+\partial f / \partial d t .
\end{aligned}
$$

Applying this to the Hamiltonian $\mathcal{H}$ itself,

$$
d \mathcal{H}\left(q_{i}, p_{i}\right) / d t=\left(\frac{\partial \mathcal{H}}{\partial q_{i}} \frac{\partial \mathcal{H}}{\partial p_{i}}-\frac{\partial \mathcal{H}}{\partial q_{i}} \frac{\partial \mathcal{H}}{\partial p_{i}}\right)=0,
$$

so $\mathcal{H}$ is the conserved energy (related to time translation invariance of the dynamics); in simple cases with kinetic energy $T(p):=\frac{1}{2} p^{2}$ and potential energy $V(q)$,

$$
\mathcal{H}(p(t), q(t))=T(p)+V(q)=\text { const }=: E .
$$

This constant relation does not imply there is no time evolution taking place: it means there is a first integral of that evolution. If there is no conserved energy, the Hamiltonian description \{(4), (5)\} does not apply. This will be the case whenever dissipative processes take place and affect the dynamics at the chosen scale of description; this occurs in a great many cases in both macro and micro physics [27.

\subsubsection{Irreversible Dynamics}

In general, friction effects mean we have an inability to retrodict if we lose information below some level of coarse graining. The simplest example is a block of mass $m$ sliding on a plane, slowing down due to constant limiting friction $F=-\mu R$ where $\mu$ is the coefficient of friction and $R=m g$ is the normal reaction, where $g$ is the acceleration due to gravity [77. The motion is a uniform deceleration; if we consider the block's motion from an initial time $t=0$, it comes to rest at some later time $t_{*}>0$. For $t<t_{*}$ the velocity $v(t)$ and position $x(t)$ of the object are given by

$$
v_{1}(t)=-\mu g t+v_{0}, x_{1}(t)=-\frac{1}{2} \mu g t^{2}+v_{0} t+x_{0}
$$

where $\left(v_{0}, x_{0}\right)$ are the initial data for $(v, x)$ at the time $t=0$. This expression shows that it comes to rest at $t^{*}=\mu g / v_{0}$. For $t>t^{*}$, the quantities $v$ and $x$ are given by

$$
v_{2}(t)=0, x_{2}(t)=X(\text { constant }),
$$


where $X:=-\frac{1}{2} \mu g t_{*}^{2}+v_{0} t_{*}+x_{0}$.

The key point now is that from the later data (14) at any time $t>t_{*}$ you cannot determine the initial data $\left(v_{0}, x_{0}\right)$, nor even the time $t_{*}$ when the object came to rest, thus you cannot reconstruct the trajectory (13) from that later data. You cannot even tell if the block came from the left or the right. The system is no longer time reversible or predictable. The direction of time is uniquely determined by the way it came to a halt (it cannot spontaneously start moving; note that the rest frame implied here is that defined by the table on which the block slides). That coming to rest was an event that took place in time; time does not cease after it comes to rest, it is the block's motion that ceases then. At macro scales, reversibility does not hold, nor is the motion predictable in both directions of time; the dynamics is not Hamiltonian.

A standard response is, if we knew how the surface was heated, we could work out where the block cane from and when it arrived. But the heat dissipates away and vanishes into thermal fluctuations (at a small enough scale, into quantum fluctuations); that record is soon irretrievably lost. The claim that "every event in the past and future is implicit in the current moment" soon ceases to be true.

\subsection{The quantum physics of the passage of time}

In the last example, our inability to predict is associated with a lack of detailed information. So if we finegrained to the smallest possible scales and collected all the available data, could we then determine uniquely what is going to happen? No, we can't predict to the future in this way because of foundational quantum uncertainty relations(see e.g. 35, 62, 53,). We cannot predict precisely when a nucleus will decay or what the velocity of the resultant particles will be, nor can we predict precisely where a photon or electron in a double-slit experiment will end up on the screen. This unpredictability is not a result of a lack of information: it is the very nature of the underlying physics.

There are two kinds of quantum evolution [62, 53, 63. First there is the unitary Schrodinger evolution

$$
i \hbar \frac{\partial|\psi\rangle}{\partial t}=\hat{H}|\psi\rangle
$$

where $|\psi(t)\rangle$ is the wave function and $\hat{H}$ the Hamiltonian operator. This equation determines its evolution: time occurs here in the same way as in classical physics, though the meaning of the relevant variable is quite different. Time rolls on and the state vector evolves; therefore probabilities change with time.

Second there is wave function reduction, associated with both state vector preparation and measurement events. Consider the wave function or state vector $|\psi(x)\rangle$. The basic expansion postulate for quantum mechanics is that before a measurement is made, $|\psi\rangle$ can be written as a linear combination of eigenstates

$$
\left|\psi_{1}\right\rangle=\sum_{n} c_{n}\left|u_{n}(x)\right\rangle
$$

where $u_{n}$ is an eigenstate of some observable $\hat{A}$ (see e.g. [53]: 5-7). Immediately after a measurement is made at a time $t=t^{*}$, however, the wavefunction is found to be in one of the eigenstates:

$$
\left|\psi_{2}\right\rangle=c_{N} u_{N}(x)
$$

for some specific index $N$. The data for $t<t^{*}$ do not determine $N$; they merely determine a probability for the outcome $N$ through the fundamental equation

$$
p_{N}=c_{N}^{2} .
$$


One can think of this as due to the probabilistic time-irreversible reduction of the wave function

$$
\left|\psi_{1}\right\rangle=\sum_{n} c_{n}\left|u_{n}(x)\right\rangle \quad \longrightarrow \quad\left|\psi_{2}\right\rangle=c_{N} u_{N}(x)
$$

([62]: 260-263). This is the event where the uncertainties of quantum theory become manifest, as the indeterminate future makes a transition to the determined past (up to this time the evolution is determinate and time reversible). Invoking a many-worlds description (see e.g. [53]) will not help determine the specific outcome: in the actually experienced universe in which we make the measurement, $N$ is unpredictable, as confirmed by experiment. The specific experimental outcome (17) that will be measured by an observer to occur at a later time is not determined by the Everett hypothesis 4

Thus the initial state (15) does not uniquely determine the final state (17); and this is not due to lack of data, it is due to the foundational nature of quantum interactions. You can predict the statistics of what is likely to happen but not the unique actual physical outcome, which unfolds in an unpredictable way as time progresses; you can only find out what this outcome is after it has happened. Furthermore, in general the time $t *$ is also not predictable from the initial data: you don't know when 'collapse of the wave function' (the transition from (15) to (17)) will happen (you can't predict when a specific excited atom will emit a photon, or a radioactive particle will decay).

We also can't retrodict to the past at the quantum level, because once the wave function has collapsed to an eigenstate we can't tell from its final state what it was before the measurement. You cannot retrodict uniquely from the state (17) immediately after the measurement takes place, or from any later state that it then evolves to via the Schrodinger equation at later times $t>t *$, because knowledge of these later states does not suffice to determine the initial state (15) at times $t<t *$ : the set of quantities $c_{n}$ are not determined by the single number $a_{N}$.

This process takes place all the time as physical events occur and have classical outcomes (in photosynthesis in plants and in nucleosynthesis in the early universe, for example); it is not necessarily associated with a measuring apparatus or the mind of an experimenter. However it is time-irreversible, causing information loss, and so is not describable by any unitary evolution. The classical world would not exist if this did not happen as an ongoing unfolding process in time.

The fact that such events happen at the quantum level does not prevent them from having macro-level effects. Many systems can act to amplify them to macro levels, including photomultipliers (whose output can be used in computers or electronic control systems). This amplification is what occurred when cosmic rays whose emission is subject to quantum uncertainty - caused genetic damage in the distant past, resulting in new phenotypes occurring [65]. The specific outcome that actually occurred was determined as it happened, when quantum emission of the relevant photons took place. Any specific emission event (a photon emission time and trajectory) was not determined by the priori quantum state, so any consequent damage to a specific gene in a particular cell at a particular time and place cannot be predicted even in principle 5 Consequently the specific evolutionary outcomes for life on Earth (the existence of dinosaurs, giraffes, humans) cannot be uniquely determined by causal evolution from detailed data at the start of life on Earth.

\footnotetext{
${ }^{4}$ This assumes that in Figure 1, all those possible paths in fact occurred; but we experience only one specific path.

${ }^{5}$ This damage is not trivial: see [71].
} 


\section{A problem: Surfaces of change}

The problem however is the claimed unique status of "the present" in the EBU - the surface where the indeterminate future is changed to the definite past at any instant. In this section I propose that there are indeed such preferred surfaces in all realistic general relativity models of space-time.

It is a fundamental feature of Special Relativity that simultaneity is not uniquely defined, it depends on the state of motion of the observer; and one presumes that in some fundamental sense the present must be regarded as a surface of constant time. What is past and future elsewhere depends on one's motion, hence the block universe model is natural: it is the only way a spacetime model can incorporate this lack of well defined surfaces of instantaneity. For different observers at a event $P$, different surfaces of simultaneity will designate different events $Q$ on a distant world line $\gamma_{1}$ as simultaneous with $P$ [34; the only resolution is that they are all simultaneous with $P$, hence time is an illusion.

However there are two fundamental points to be made here that completely change the picture. First, the physical events that shape how things evolve are based on particle interactions, and take place along timelike or null world lines, not on spacelike surfaces, which are secondary. The concept of simultaneity is only physically meaningful for neighboring events; it has no physical impact for distant events, it is merely a theoretical construct we like to make in our minds. What we think is instantaneous makes no difference to our interaction with a vehicle on Mars. What is significant is firstly what happens over there, secondly what happens here on Earth, and, thirdly the signals between us. Simultaneity does not enter into it.

What really matters is proper time $\tau$ measured along timelines $x^{i}(v)$, determined from the metric tensor $g_{i j}\left(x^{k}\right)$ by the basic formula [49, 34.

$$
\tau=\int \sqrt{-d s^{2}}=\int \sqrt{-g_{i j}\left(d x^{i} / d v\right)\left(d x^{j} / d v\right)} d v
$$

Indeed this is the reason why the metric tensor is central to relativity theory: as well as determining which lines are null lines ( $d \tau=0$ all along the curve), it determines proper time along timelike world lines. Natural surfaces of constant time are given by this integral since the start of the universe. Thus we can propose that

The present: The ever-changing surface $S(\tau)$ separating the future and past - the 'present' at the time $\tau$ is the surface $\{\tau=$ constant $\}$ determined by the integral (20) along a family of fundamental world lines starting at the beginning of space time.

(if the universe existed forever we have to start at some arbitrarily chosen 'present' time $\left\{\tau_{0}=\right.$ const $\}$, which we assume exists, and integrate from there).

But is this well defined, given that there are no preferred world-lines in the flat spacetime of special relativity? The second fundamental feature is that it is general relativity that describes the structure of space time, not special relativity. Gravity governs space-time curvature [4], and because there is no perfect vacuum anywhere in the real universe (inter alia because cosmic blackbody background radiation permeates the Solar System and all of interstellar and intergalactic space [18), space time is nowhere flat or even of constant curvature; therefore there are preferred timelike lines everywhere in any realistic spacetime model [21. The special relativity argument does not apply.

A unique geometrically determined choice for fundamental worldlines is the set of timelike eigenlines $x^{a}(v)$ of the Ricci tensor (they will exist and be unique for all realistic matter, because of the energy conditions such matter obeys [49]). Their 4 -velocities $u^{a}(v)=d x^{a}(v) / d v$ satisfy

$$
T_{a b} u^{b}=\lambda_{1} u_{a} \Leftrightarrow R_{a b} u^{b}=\lambda_{2} u_{a}
$$


where the equivalence follows from the Einstein field equations. Thus we can further propose that

Fundamental world lines: the proper time integral (20) used to define the present is taken along the world lines with 4-velocity $u^{a}(v)$ satisfying (21).

In effect this is the proper time comoving gauge used in perturbation theories: it will of course give the usual surfaces of constant time in the standard Friedmann-Lemaitre-Robertson-Walker (FLRW) cosmologies.

Two key issues arise regarding this proposal:

- What about general covariance and local Lorentz invariance? These are symmetries of the general theory, not of its solutions. Interesting solutions break the symmetries of the theory: this is not surprising, as we know that broken symmetries are the key to interesting physics [3].

- What about simultaneity? In general these surfaces are not related to simultaneity as determined by radar [34; indeed this is even so in the FLRW spacetimes (where the surfaces of homogeneity are generically not simultaneous, according to the radar definition [29]). The flow lines are not necessarily orthogonal to the surfaces of constant time; indeed they may have non-zero vorticity and acceleration as well as shear and expansion, so there may be no surfaces orthogonal to the flow lines [21]. More than that: the surfaces determined in this way are not even necessarily spacelike, in an inhomogeneous spacetime.

The latter feature means that the there may possibly be a time horizon: a null boundary where these surfaces make a transition from spacelike to timelike. This will of course only happen for very large gravitational fields such as occur in black holes, indeed these surfaces may well usually coincide with an event horizon. The initial value problem will then be very different when based in data in these surfaces; however even if these surfaces become timelike (necessarily then being null in some places), data on them will still determine the spacetime in their future and past Cauchy development, up to intersections of this development with surfaces where the outcome is already determined. The physics of time then will be quite different than usual: this needs investigation. It could relate to the black hole information paradox.

In summary: While the general coordinate invariance invoked in general relativity theory might be thought to proclaim there are no preferred such surfaces, in any particular solution this is not the case there will be preferred timelike lines in any realistic cosmological solution. The result will be existence of a family of preferred surfaces representing constant proper time $\tau$ since the start of the universe along these fundamental world lines. The proposal is that each represents what was the "present" at the corresponding time $\tau$, for all times up up to the present time $\tau_{0}$ (they don't exist for $\tau>\tau_{0}$, for that spacetime is not yet determined). These surfaces are derivative rather than primary, as they result from the configuration of fundamental world lines. They will usually not be instantaneous as determined by radar soundings.

\section{Other Arguments against an EBU}

A series of other arguments, both physical and philosophical, have been deployed in favour of the standard Block Universe picture [72, and hence deny the EBU proposal. In this section I review them, and argue that none of them are fatal. 


\subsection{Categorization problem}

A philosophical argument is that the past, present, and future are exclusive categories, so a single event can't have the character of belonging to all three. The counter is as follows:

Suppose E happens at $t_{E}$.

At time $t_{1}<t_{E}, \mathrm{E}$ is in future,

At time $t_{1}=t_{E}, \mathrm{E}$ is in present,

At time $t_{1}>t_{E}, \mathrm{E}$ is in past.

Its category changes - that is the essence of the flow of time - so this is a semantic problem, not a logical one. One needs adequate semantic usage and philosophical categories to allow description of this change: language usage can't prevent the flow of time.

\subsection{Not necessary to describe events}

Davies [17] and Rovelli 69] claim time does not flow because it's not needed to describe the relations between relevant variables, which are all that matter physically. Thus you can always get correlations between position $p(t)$ and momentum $q(t)$ for a system by eliminating the time variable: solve for $t=t(q)$ and then substitute to get $p(t)=p(t(q))=p(q)$, and time has vanished! For example in the case of the simple harmonic oscillator (equation (2)), this gives the SHO phase plane:

$$
q^{2}+(p / m)^{2}=A^{2}
$$

Thus one can describe system changes by relating component variables to one another, rather than to a global idea of time; which suggests nothing happens or changes, they are just correlated.

Yes indeed, one can find this time-independent representation of what happens 6 But that does not mean that time does not flow, it just means that the results of times flow are correlations between relevant variables. That abstraction represents part of what happens, namely the relation between $p$ and $q$, and omits other parts, namely the relation to time. One can put time back to get

$$
q(t)^{2}+(p(t) / m)^{2}=A^{2}, q(t)=A \cos (\omega t-\phi)
$$

and the point representing the system moves along the flow lines as time changes. The first model leaves out part of what is happening: that does not mean it does not happen, it just means it's a partial model of reality, including some aspects and omitting others. It leaves out the way that the continually changing correlations flow smoothly one after another in a continuous ongoing way.

\subsection{Rates of change}

A key question is, "What determines the rate of flow of time?", or "How fast does time pass?" Davies and others suggest there is no sensible answer to this question. In contrast, I claim that the answer is given by equation (20), which determines proper time $\tau$ along any world line. This is the time that will be measured along that world line by any perfect clock [49, 34]; real world clocks - oscillators that obey the Simple Harmonic equation - are approximations to such ideal clocks, and it is the relation between such clocks and other physical events that measures the passage of time.

\footnotetext{
${ }^{6}$ This is just the energy integral (12).
} 
The preferred time parameter The whole edifice of physics is built on the assumption that we can build such clocks to a good approximation, giving a time parameter $\tau$ that appears equally in all dynamical equations of physics: Newton's equation of motion [36, Maxwell's equations [37, the Schrödinger equation [38, General Relativity expressed in a 1+3 covariant formalism [21, and so on; because of this, a lot of money is spent on building idealized clocks that are understood to be more accurate than any previous clock 7 . Standard physics would not work if you needed a different time parameter in each of these equations. Special and general relativity identify that time as proper time (20) along timelike worldlines.

Given such clocks, the rate of change with time of any variable $f(\tau)$ along a world line is given by

$$
f^{\prime}=d f / d \tau \text {. }
$$

Then choosing $f(\tau)=\tau$, the answer to the question posed is that the rate of change of time is unity:

$$
\tau^{\prime}=d \tau / d \tau=1
$$

In other words, through (20) the rate of change of time in any particular coordinate system is determined by the metric tensor. Using normalised comoving coordinates with $u^{a}=\delta_{0}^{a}$ and the time parameter $v$ chosen as $\tau$ [21], $g_{00}=-1$ and (20) becomes

$$
\tau=\int d \tau
$$

The relative flow of time along different world lines may be different: that is the phenomenon of time dilation, caused by the varying gravitational potentials represented by the metric tensor [78. But this does not mean it is not well defined along each world line.

The metric evolution So if the metric tensor determines proper time, what determines the metric tensor? The Einstein field equations, of course 49. These can be expressed in many ways, for example a 1+3 covariant formalism [21, a tetrad formalism [20, or the ADM formalism [5]. Following the ADM approach, the first fundamental form (the metric) is represented as

$$
d s^{2}=\left(-N^{2}+N_{i} N^{i}\right) d t^{2}+N_{i} d x^{i} d t+g_{i j} d x^{i} d x^{j}
$$

where $i, j=1,2,3$. The lapse function $N\left(x^{\alpha}\right)$ and shift vector $N_{i}\left(x^{\beta}\right)$ represent coordinate choices, and can be chosen arbitrarily; $g_{i j}\left(x^{\alpha}\right)$ is the metric of the 3 -spaces $\{t=$ const $\}$. The second fundamental form is

$$
\pi_{i j}=n_{i ; j}
$$

where the normal to the surfaces $\{t=$ const $\}$ is $n_{i}=\delta_{i}^{0}$; the matter flow lines have tangent vector $u^{i}=\delta_{0}^{i}$ (which differs from $n^{i}=g^{i j} n_{j}$ whenever $N_{i} \neq 0$, cf [28]). The field equations for $g_{i j}$ are as follows (where 3 -dimensional quantities have the prefix (3)): four constraint equations

$$
\begin{aligned}
{ }^{(3)} R & +\pi^{2}-\pi_{i j} \pi^{i j}=16 \pi \rho_{H}, \\
R^{\mu} & :=-2 \pi^{\mu j}{ }_{\mid j}=16 \pi T_{0}^{\mu}
\end{aligned}
$$

\footnotetext{
${ }^{7}$ See e.g. http://en.wikipedia.org/wiki/Clock accurate navigation for example requires accurate timekeeping [75], which is thus a core feature of GPS systems.
} 
where " $\mid j$ " represents the covariant derivative in the 3 -surfaces, and twelve evolution equations

$$
\begin{aligned}
\partial_{t} g_{i j} & =2 N g^{-1 / 2}\left(\pi i j-\frac{1}{2} g_{i j} \pi\right)+N_{i \mid j}+N_{j \mid i}, \\
\partial_{t} \pi_{i j} & =-N g^{-1 / 2}\left({ }^{(3)} R_{i j}-\frac{1}{2} g_{i j}{ }^{(3)} R\right)+\frac{1}{2} N g^{-1 / 2} g_{i j}\left(\pi_{m n} \pi^{m n}-\frac{1}{2} \pi^{2}\right)-2 N g^{-1 / 2}\left(\pi^{i m} \pi_{m}^{j}\right. \\
& \left.-\frac{1}{2} \pi \pi^{i j}\right)+\sqrt{g}\left(N^{\mid i j}-g^{i j} N_{\mid m}^{\mid m}\right)+\left(\pi^{i j} N^{m}\right)_{\mid m}-N_{\mid m}^{i} \pi^{m j}-N_{\mid m}^{j} \pi^{m i}+16 \pi \hat{T}_{i j} .
\end{aligned}
$$

Equations of state for matter must be added, and the matter conservation equations $T_{; b}^{a b}=0$ satisfied (as is required for consistency of the evolution equations). Then (31) determines the rate of change of the metric $g_{i j}\left(x^{\alpha}\right)$ relative to the ADM time coordinate; (32) determines the rate of change of the geometric source terms $\pi_{i j}$ occurring in (31); the matter equations determine the rate of change of the matter terms. How this works out in practice is shown in depth in 4. Overall this determines the metric tensor as a function of time, and hence evolution of the surfaces of constant time as defined above (which are determined by the metric).

This can be worked out using any time surfaces (that is the merit of the ADM formalism); in particular one can specialise the time surfaces and flow lines to those defined above (\$3):

1. We choose the 4-velocity to be a Ricci Eigenvector:

$$
T_{0}^{\mu}=0 \Rightarrow R^{\mu}=-2 \pi_{\mid j}^{\mu j}=0,
$$

which algebraically determines the shift vector $N_{i}\left(x^{j}\right)$, thereby solving the constraint equations (30);

2. We determine the lapse function $N\left(x^{i}\right)$ by the condition that the time parameter $t$ measures proper time $\tau$ along the fundamental flow lines.

These conditions uniquely determine the lapse and shift (see the Appendix for details). Then, given the equations of state and dynamical equations for the matter (in the case of a perfect fluid, equations (45)-(47) on page 45 of [4]) with $S^{\kappa}=S \delta_{0}^{\kappa}$, equations (31), (32) determine the time evolution of the metric in terms of proper time $\tau$ along the fundamental flow lines; the constraints are conserved because of energy-momentum conservation. The development of spacetime with time takes place just as is the case for other physical fields, with the relevant time parameter being proper time along the fundamental flow lines. There is no problem with either the existence or the rate of flow of time.

Predictability: Do these equations mean the spacetime development is uniquely determined to the future and the past from initial data? That all depends on the equations of state of the matter content: the relations between the density $\rho_{H}$ in (29), pressure tensor $\hat{T}_{i j}$ in (32), and momentum density $T_{0}^{\mu}$ in (30). These quantities depend on the frame chosen, and $T_{0}^{\mu}$ is zero when we make the choice (33).

Assuming this choice, define the pressure $p$ and anisotropic stress $\Pi_{i j}$ by

$$
p=\frac{1}{3} g^{i j} \hat{T}_{i j}, \Pi_{i j}=\hat{T}_{i j}-p g_{i j}
$$

The future and past will be uniquely determined for simple equations of state such as non-interacting baryons plus radiation, as in standard cosmological models:

$$
\rho_{H}=\rho_{b}+\rho_{r}, p=p_{b}+p_{r}=\frac{1}{3} \rho_{r}, \Pi_{i j}=0
$$

where the energy density conservation equations will determine the time evolution of $\rho_{b}, \rho_{r}$. However 
- one can have dissipative processes (shear viscosity or bulk viscosity, for example [21]), so the evolution is not time reversible - a Hamiltonian description does not apply to the matter, and hence also does not apply to the combined (matter, gravity) system, where matter determines space-time curvature;

- one can have an explicitly time dependent equation of state:

$$
p=p\left(\rho_{H}, \tau\right), \Pi_{i j}=\Pi_{i j}\left(\rho_{H}, \tau\right)
$$

and predictability is no longer the case if the time dependence is not predictable from the available initial data at the relevant scales; again a time-reversible and predictive Hamiltionian description can't be used for the system as a whole.

For example one can have a massive body where effectively one has

$$
\Pi_{i j}(\tau)=F(\tau) \Pi_{i j}(0)
$$

where $F(\tau)$ represents internal dynamics not visible to the external world 8 ; there might be a mechanism here that is computer controlled via an algorithm embodying a random number generator 9 or based in random signals generated via radioactive decay [56. Then as explained above (\$2 and Fig,1), the initial data do not determine the outcome $(F(\tau)$ indicates a causal influence but not a predictable functional relation). One can only determine what will happen as it happens.

So equations (31), (32) determine the time evolution of the spacetime, but do not guarantee predictability either to the future or the past. That depends on the physics of the matter; if quantum unpredictability gets amplifed to macro scales, the spacetime evolution is intrinsically undetermined till it happens (as mentioned above, this was essentially what happened during the generation of seed inhomogeneities in the inflationary era in the very early universe).

Conclusion: time flows at the rate of one second per second, with the metric tensor determining what this rate is for clocks and every other physical system (the choice of units is of course arbitrary, but can be done in a way that makes sense). The result is that clock readings and particle motions are correlated in a way that enable us to reliably predict motions. So yes, such correlations are fundamental to our experience of the flow of time (as emphasized by Davies); they are a result of its inexorable omnipresent continuing flow equally in all physical systems. Can you change the rate of time? No! Can you stop time? No! Can you reverse time? No! Like Old Man River, it just keeps flowing on; that is the primitive expressed in all the time evolution equations of physics [36, 37, 38, 21] and the related existence and uniqueness theorems [49.

\subsection{Time parameter invariance of General Relativity}

What about the time parameter invariance of General Relativity, as made manifest in the ADM formalism [5, 41]? This has basically already been dealt with in the discussion above:

- The gravitational side of the ADM equations may be time-parameter invariant, but the matter side is not, in particular because rescaling time changes the value of the kinetic energy $T(p)$ while leaving the potential energy $U\left(x^{i}\right)$ unchanged. Hence any solutions with matter present (i.e. all realistic solutions) will not be time parameter invariant; this is part of the ongoing tension between the geometric and matter sides of the Einstein Field Equations;

\footnotetext{
${ }^{8}$ Bondi's massive objects Tweedledum and Tweedledee incorporated this idea: see Narlikar [61] for a description.

${ }^{9}$ see http://www.random.org/randomness/ for a discussion.
} 
- This is part of the broader theme mentioned above: specific solutions of the theory have less symmetry than the theory itself; this symmetry breaking is a key feature of all realistic solutions of the equations of physics [3], and in particular cosmological solutions (\$3);

- Proper time (20) along fundamental world lines (21) provides a preferred time parameter in realistic solutions of general relativity theory.

Local physics does indeed have a preferred time parameter: for example in a Simple Harmonic Oscillator using standard time $t, q(t)=A \cos (\omega t-\phi)$ (see (2)); these cycles measure time $t$ like a metronome (which is why SHO's are used as clocks). One can in principle change to an arbitrary time $t^{\prime}$,

$$
t^{\prime}=t^{\prime}(t) \Rightarrow t=t\left(t^{\prime}\right) \Rightarrow q=A \cos \left(\omega t\left(t^{\prime}\right)-\phi\right),
$$

for example

$$
t^{\prime}=\exp H(t) \Rightarrow t=\exp \left(-H t^{\prime}\right) \Rightarrow q=A \cos \left(\omega \exp \left(-H t^{\prime}\right)-\phi\right)
$$

hence the regular motion no longer is represented as regular. This is because one has chosen a peculiar time, which will not correlate simply with any other physical behaviour either (41]:57-60). The sensible choice of time is that which makes sense of patterns of physics behaviour; so the maximum sensible variation is $t^{\prime}=\alpha t+\beta$ (just as in the case of the allowed change in affine parameter along geodesics). One can choose any other reparametrisation of time; but any except affine transformations of proper time confuse and hide what is actually happening. One is able to choose proper time, and does so if one wants to illuminate the physics.

It is non-optimal to examine the dynamics of general relativity without acknowledging the central role of the metric tensor $g_{i j}$ and resultant proper time (20) along world lines - which are at the core of the physical interpretation of General Relativity [49, 34. You can use a proper time coordinate $\tau$ in the ADM formalism (as shown above in 4.2 , this is a general way one can solve the problem of time in ADM dynamics, see [66]). That choice ties this time parameter in to the rest of physics, and in particular to time as measured by local ideal clocks (such as a cesium atom). Consequently the flow of time is then characterised by the relation between such clocks and other physical events, including the gravitational dynamics represented by the ADM evolution equations (31), (32). In this sense time is relational (cf: [41]:163-166).

In the case of the standard FLRW models of cosmology, the usual metric is

$$
d s^{2}=-d \tau^{2}+a^{2}(\tau) d \sigma^{2}
$$

where $d \sigma^{2}\left(x^{i}\right)$ is a time-independent 3-space of constant curvature [21, 18, and $\tau$ is precisely the preferred time coordinate defined above (\$3), as the flow lines $u^{a}=\delta_{0}^{a}$ are Ricci flow lines and $\tau$ is proper time along them (these are normalized comoving coordinates [21]). Just as in the case of the simple harmonic oscillator, one can choose arbitrary other time parameters - but it is very perverse to do so, and this is not done in practice, except for one other common choice: use of a conformal time parameter $\eta(\tau)$ :

$$
\eta=\int d \tau / a(\tau) \Rightarrow d s^{2}=a^{2}(\eta)\left(-d \eta^{2}+d \sigma^{2}\right)
$$

which is a very poor representation of proper time but represents causal structure very well [49, 34. However this still has the same surfaces of constant time as in (40), and is always acknowledged to be different from the proper time $\tau$ that is fundamental in local physics. One never finds proposals for a time $t=t\left(\tau, x^{i}\right)$ with $\partial t / \partial x i \neq 0$, which would have different surfaces of constant time. The usual choice as in (40), agreeing with 
the proposal made here in $\S(3)$, ties time $\tau$ in to all the rest of physics, astronomy, geology, technology, and biology.

In conclusion: Standard physics is based in choice of a preferred time parameter $\tau$ along matter world lines. General Relativity both allows such a choice, and can itself be written in terms of that choice.

\section{Time with an underlying timeless substratum}

There are a number of proposals for an effective time to emerge somehow in the context of a timeless substratum. These include the Mott proposal (\$5.1), the Rovelli proposal (\$5.2), and proposals based in the the Wheeler-de Witt equation (\$5.3). In this section I will comment on them in turn.

As a preliminary, I first remark that there are two common themes cutting across them all:

- If an effective time emerges at the macro scale, then however that happens, it emerges: and the EBU proposal is then good at macro scales, no matter how it relates to a timeless substrate;

- All of these approaches are based on unitary Schrödinger evolution, so none of them effectively tackles the non-unitary evolution associated with both state vector preparation and quantum measurements [53. Hence they are omitting a key way that a flow of time takes place at the micro level $(\$ 2.3), 10$

I regard the latter as a particularly significant problem for all such proposals (cf. [26]).

\subsection{Interaction with the environment}

Mott [60] and Briggs and Rost [9, 10] suggest that the Time Independent Schrodinger Equation ('TISE') is more fundamental than the Time Dependent Schrodinger Equation (15) ('TDSE'): indeed that the latter emerges from the former, the interaction between parts in a timeless whole generates an effective time characterizing interaction between the parts. This is summarised in [10] as follows:

"Following work of Born, in 1931 Mott(3) described the impact of $\alpha$-particles on atoms by treating both atom and beam quantum-mechanically with the TISE. Then he showed that for a high energy beam he could describe its motion classically resulting in a time-dependent Hamiltonian and TDSE for the atom alone ... time is entering only from a classical interacting environment .. time enters the quantum Hamiltonian only when some external system is approximated by classical behaviour".

Thus this is an emergence of time by a top-down interaction from the environment.

According to Briggs and Rost [10, one can use the TIDE in the form

$$
H \Psi=E \Psi \Leftrightarrow\left(H_{\mathcal{E}}+H_{\mathcal{S}}+H_{\mathcal{I}}\right) \Psi=E \Psi
$$

where $\mathcal{E}$ represents the environment, $\mathcal{S}$ the system and $\mathcal{I}$ their interaction. Through a kinetic energy term, the interaction Hamiltonian somewhat mysteriously introduces an effective time into the wave function for the system. If one accepts this proposal, it is a way that an effective time variation is induced at the quantum level due to top-down effects from the environment - a proposal that is in consonance with the broad suggestions of the effectiveness of top-down effects in quantum physics presented in [26]. Once this has occurred, one has an effective EBU situation at the micro as well as the macro level.

\footnotetext{
${ }^{10}$ The many worlds view often associated with the Wheeler-de Witt equation proposes to deal with measurements, but not with state vector preparation, which is also non-unitary [26].
} 


\subsection{Get it by coarse graining?}

By contrast Rovelli [69] suggests time can emerge in a bottom-up way from a timeless substrate as a thermodynamic variable. I find it difficult to see how any process of coarse graining a static state can introduce time, but in any case this proposal faces two other problems.

First, it is based in the idea of equilibrium distribution: "Whatever the statistical state $\rho$ is, there exists always a variable $t_{\rho}$, measured by the thermal clock, with respect to which the system is in equilibrium and physics is the same as in the conventional nonrelativistic statistical case!" However equilibrium is a state that emerges through molecular collisions: if there is no time there will be no such collisions, and no reason whatever to assume that equilibrium is the most probable state of the system. This proposal embodies a hidden assumption that time already exists.

Second, it is based on the underlying symmetries of Hamiltonian dynamics: "mechanics does not single out a preferred variable, because all mechanical predictions can be obtained using the relativistic hamiltonian $H$, which treats all variables on equal footing". But this symmetry applies particularly to the direction of time: Hamiltonian dynamics (4), (5) has a time-reversal symmetry

$$
x \rightarrow x, t \rightarrow-t, q \rightarrow q, p \rightarrow-p,
$$

hence like every other proposal for the purely bottom up emergence of time, it has problems determining the arrow of time. An initial reaction to any such proposal is that coarse graining from micro to macro scales convincingly results in an arrow of time, as shown beautifully by Boltzmann's H-Theorem (85]:43-48), resulting from the fact that random motions in phase space takes one from less probable to more probable regions of phase space $([63]: 686-696 ;$ [2]:43-47; 64]:9-56). Hence one can show that entropy increases to the future; the second law of thermodynamics at the macro level emerges from the coarse grained underlying micro theory. The quantum theory version of this result is the statement that the density matrix of open system evolves in a time asymmetric manner, leading to an increase in entropy ([8]:123-125).

But this apparent appearance of an arrow of time from the underlying theory is an illusion, as the underlying theory is time symmetric, so there is no way an arrow of time can emerge by any local coarse graining procedure. Indeed the derivation of the increase of entropy in Boltzmann's H-Theorem applies equally to both directions of time ( paradox (62]: Fig 7.6; 63]:696-699; 64]):

Time symmetry of the H-Theorem: Boltzmann's H-theorem predicts entropy will increase to both the future and the past.

The same will apply to the quantum theory derivation of an increase of entropy through evolution of the density matrix ([8]:123-125, [42]:38-42, 53-58): it cannot resolve where the arrow of time comes from, or indeed why it is the same everywhere. The latter is a key question for any local proposal for determining the arrow of time:

The arrow of time locality issue: If there is a purely local process for determining the arrow of time, why does it give the same result everywhere?

We are unaware of any contradictions as regards the direction of the arrow of time in the universe around us, either locally (time does not run backwards anywhere in Earth) or astronomically (irreversible process in distant galaxies seem to run in the same direction of time as here [68]). Some top-down coordinating

mechanism is called for to guarantee the future direction of time will be the same everywhere; that is lacking in any purely bottom-up proposal, which is by its nature based in local interactions only. 
Kupervasser et al [58] suggest that interaction between two subsystems with a different arrow of time will cause a decay towards a universal direction for the arrow of time. This is a very interesting claim, but has two problems: first, how can a coherent interaction take place at an interface where the direction of time is different on the two sides? It seems a priori that paradoxical behaviour will abound, as closed causal loops will necessarily occur there. And if this does work despite these problems, then one has to show that there is sufficient time available since the start of the universe that all domains opposite to the dominant one get coerced to join the majority; effective causal horizons [49] might even prevent this occurring. Kupervasser et al do not put their proposal in the cosmic context, so this too is unresolved.

\subsection{The Wheeler de Witt equation}

A whole literature on the problem of time in quantum cosmology [45, 7, 15] suggests that an effective time emerges from a time-independent wave function of the universe determined by the Wheeler-de Witt equation [46, 47, 13. As stated by Hartle [46,

"In quantum mechanics, any system - the universe included - is described by a wave function $\Psi$. There is a local dynamical law called the Schrödinger equation that governs how the wave function changes in time:

$$
i \hbar \frac{d|\Psi(t)\rangle}{d t}=H|\Psi(t)\rangle \quad \text { (dynamical law) }
$$

Here the operator $\mathrm{H}$, the Hamiltonian, summarizes the dynamical theory.... the Schrödinger equation doesn't make any predictions itself, it requires an initial condition. This is

$$
|\Psi(0)\rangle \quad \text { (initial condition) }
$$

When we consider the universe as a quantum mechanical system, this initial condition is Hawking's wave function of the universe [47."

This is the basis of quantum cosmology [43.

The problem of time then arises because in the case of General Relativity, where $|\Psi\rangle$ is a function of 3-geometries: $|\Psi\rangle=\left|\Psi\left(h_{i j}\right)\right\rangle$, the Hamiltonian is such as to lead to the Wheeler-de Witt equation for the wave function of the universe:

$$
H|\Psi\rangle=0 .
$$

So by (44), $|\Psi\rangle$ is time independent and the probabilities for quantum outcomes in the universe, which are expressed by the wave function $|\Psi\rangle$, are unchanging in time (this is a simplified sketch; for details, see [45, 2, 50]). Hence time does not pass, the universe just is. Time is an illusion [7. A large literature then tries to show how an effective time can emerge from this timeless context [45, 7, 15]. I will make four points.

First, Arnowitt, Deser and Misner write of the Hamiltonian formalism as follows [5]:

Since the relation between $q_{M+1}$ and $\tau$ is undetermined, we are free to specify it explicitly, i.e., impose a "coordinate condition". If, in particular, this relation is chosen to be $q_{M+1}=\tau$ (a condition which also determines $N$ ), the action (2.4) then reduces [to] (2.5) with the notational change $q_{M+1} \rightarrow \tau$; the non-vanishing Hamiltonian only arises as a result of this process. 
This is the choice made above (44.3); the corresponding Hamiltonian will be non-zero as indicated in this quote, so (46) will not hold (cf. 66]).

Second, is there an alternative proposal that does not lead to (46)? Yes indeed: unimodular gravity (which produces a trace free version of the Einstein Field Equations [33]) has the same effective gravitational equations as General Relativity Theory $[82$ but makes $H \neq 0$ and so solves the time problem of quantum cosmology. Smolin [73] states this as follows: "Sorkin [76] and Unruh [80, 81] have pointed out that unimodular gravity has a nonvanishing Hamiltonian and hence evolves quantum states in terms of a global time given by an analogue of the Schrodinger equation". This removes the basis of the problem.

It has other major benefits: as emphasized by Weinberg [82 and Smolin [73, it also solves the strong cosmological constant problem: the discrepancy factor of at least $10^{70}$ between estimates of the vacuum energy density and the cosmologically determined value of the cosmological constant 18. This resolution is a crucial need in relating quantum field theory to general relativity: it is a sine qua non for consistent physics, hence

Reconciling General Relativity and Quantum Field Theory: evidence from cosmology [18] of the small size of the cosmological constant strongly favours the trace-free version of the Einstein Equations over the usual version [33].11]

Then we can have (44) without (46).

Third, this analysis assumes a Hamiltonian evolution (44) holds for the wave function of the universe as a whole at all times. This is sometimes justified by saying that as there can be no external measuring apparatus for the universe as a whole to interact with, no measurement or wave function collapse can take place: only unitary evolution will occur. However one can advocate an emergent view of higher level laws of causation from lower level physical interactions [26]. On this view, the wave function of the universe $\left|\psi_{U}\right\rangle$ is the wave function obtained by composition of all its components: it is a sum of terms of the form

$$
\left|\psi_{U}\right\rangle=\left|\psi_{1}\right\rangle \otimes\left|\psi_{2}\right\rangle \otimes \ldots \otimes\left|\psi_{N}\right\rangle
$$

where $N$ is the number of constituents making up the universe and $\left|\psi_{i}\right\rangle$ the wave function for degrees of freedom of the $i t h$ component. This will not evolve unitarily if any wave function collapse takes place for any component anywhere in the universe. But measurements do take place and classical physics does emerge. Thus on this view, we are not entitled to assume existence of a wave function for the entire universe that always obeys (46): this starting assumption is unjustified, at least at recent times.

Finally, the argument above is centrally based on the Wheeler de Witt equation, but it is not a tested and proven part of physics:

Missing Confirmation: we have no observational or experimental evidence that the Wheeler de Witt equation in fact describes the evolution of the real universe at any time.

It is an untested extrapolation of known physics, which extrapolation may or may not represent reality adequately [12. Actually one can suggest that everyday experience strongly suggests it is not true (this is hinted at in [50]). That is the topic of the next section.

\footnotetext{
${ }^{11}$ If one applies Ockham's razor ("entities must not be multiplied beyond necessity)" the proposed multiverse solution is severely disfavored in comparison with this resolution of the issue, which does not involve an infinitude of unobservable entities.
} 


\section{$6 \quad$ It's all in the mind}

Barbour [7] tackles the key issue of how the mind can experience the passing of time in the timeless context of the Wheeler de Wit equation: how do we reconcile the conclusion that time is an illusion with the fact we do indeed experience the passage of time? This section reviews that suggestion, and argues that it is fatally flawed; and that this issue is a major problem for all proposals that time is an illusion.

Barbour claims 17, 12 there exist records of events that our brains read sequentially, and so create a false illusion of the passage of time. Thus brain processes are responsible for illusion of change. But "processes" are things that unfold in time! - there are no processes unless time flows. You can't perceive a flow of time unless time flows, because perception is a process that takes place in time.

The prevalent view of present day neuroscience [55] is that mental states $\Phi$ are functions of brain states $B$ which are based in the underlying neuronal states $b_{i}$, determined by genetics, chemistry, and physics interactions in the brain, taking place in the overall physical, social and psychological environment $\mathcal{E}$. Thus

$$
\Phi=\Phi(B)=\Phi\left(b_{i}, \mathcal{E}\right) .
$$

If time does not flow in microphysics, in a given unchanging environment

$$
\left\{d b_{i} / d t=0, d \mathcal{E} / d t=0\right\} \Rightarrow d \Phi / d t=0:
$$

mental states cannot evolve, unless they are driven in some mysterious unspecified way by changes in the the environment: but on Barbour's argument, that too cannot evolve.

But one thing we do know is that time does flow in our experience (indeed 'knowing' is a key part of that experience!). Hence the assumption that time does not flow in the underlying microphysics cannot be true: the data proves it to be wrong. If Barbour's view is correct and no physical events take place, then - as the brain is based in physics - no such record-reading processes can take place. Rather than showing time is an illusion, I suggest the implication runs the other way:

Taking everyday life seriously: comparing the conclusion ('time is an illusion') with the evidence from mental life, by (49) the contradiction between them is proof the WdeW equation (46) does not apply to the universe as a whole at the present time, as proposed by Barbour.

If there is a meaningful wave function of the universe (perhaps defined by (47)), it does not evolve in a unitary way. The great merit of Barbour's book is that it takes the Wheeler de Witt equation seriously, and pursues the implications to their logical conclusion; the evidence from daily life then shows it to be wrong.

A further set of issues arise as regards the perception of time. Experienced time $\tau_{\text {exp }}$ is a function of proper time $\tau$ but also on the emotional and psychological context. It also has a minimal resolution $\tau_{\min }$ because of the interaction times in the brain: we cannot distinguish events at smaller times scales because our senses necessarily average over micro times scales with a window function $W\left(\tau_{\min }\right)$. But all this is unrelated to the fundamental issues of the nature of time we are dealing with here: it is to do with brain functioning. The brain is not necessarily a good clock, and it will not click over instantaneously: there will be a finite width to its time resolution. But the fact it works as it does is evidence of the flow of time in physics, because the brain is based in physics. It's not just a series of correlations: it's an ordered sequence of causally related correlations that flow from each other in an ongoing process enabling mental life. 


\section{Taking delayed choice quantum effects into account}

This paper so far is based on a classical view of physics. The EBU proposal made so far does not take into account the delayed choice experiments of quantum theory, which suggest one can in some circumstances "reach back into the past" to affect things there. This section briefly comments on how one can extend the EBU model to take this feature into account.

One can extend the EBU view to one that takes account of this aspect of quantum physics through proposing a "Crystallizing Block Universe" (CBU), where "the present" is effectively the transition region in which quantum uncertainty changes to classical definiteness [30. Such a crystallization, however, does not take place simultaneously, as it does in the simple classical picture. Quantum physics appears to allow some degree of influence of the present on the past, as indicated by such effects as Wheeler's delayed choice experiments [83, 54] and Scully's quantum eraser [57]. (see the summaries of these effects in [1, 44]).

The CBU picture is an extension of the EBU where local events may lead or lag the overall flow of time, thus allowing some apparent influences from the future to the past as evidenced in those experiments. It adequately reflects such effects by distinguishing the transitional events where uncertainty changes to certainty, which may in some cases be delayed till after the apparent "present time."

I have not here tried to relate the EBU picture to the issue of entanglement and EPR type of experiments [1, 44. The way those experiments relate to simultaneity and the flow of time as proposed here is unclear; this is a topic for future research.

\section{The arrow of time and closed time like lines}

Two closely related problems are the arrow of time problem, and the issue of closed timelike lines. This section discusses how the EBU proposal reformulates the first issue, and solves the second.

\subsection{The Arrow of time}

As regards the arrow of time problem [16, 85, if the EBU view is correct, the Wheeler-Feynman prescription for introducing the arrow of time by integration over the far future [84, and associated views comparing the far future with the distant past [62, 31, 23, are invalid approaches to solving the arrow of time problem, for it is not possible to do integrations over future time domains if they do not yet exist. Indeed the use of half-advanced and half-retarded Feynman propagators in quantum field theory then becomes a calculational tool representing a local symmetry of the underlying physics that does not reflect the nature of emergent physical reality, in which that symmetry is broken.

The arrow of time problem in this EBU context is revisited in a companion paper [27]. The key point is that

The direction of time: The arrow of time arises fundamentally because the future does not yet exist: a global asymmetry in the physics context. The Feynman propagator can only be integrated over the past, as the future spacetime domain is yet to be determined.

One can be influenced at the present time from many causes lying in our past, as they have already taken place and their influence can thereafter be felt. One cannot be influenced by causes coming from the future, for they have not yet come into being. The history of the universe has brought the past into being, which 
is steadily extending to the future, and the future is just a set of unresolved potentialities at present. One cannot integrate over future events to determine their influence on the present not only because they do not yet exist, but because they are not even determined at present (\$2).

The direction of the arrow of time is thus determined in a contingent way in the EBU context [27]: it is the direction of time leading from what already has come into existence (the past) to the present. Collapse of the quantum wave function is a prime candidate for a location of a physical solution to the coming-into-being problem, and manifests itself as a form of time-asymmetric top-down action 25] from the universe as a whole to local systems (cf. 62]). A key further feature is that the initial state of the universe was very special with very low entropy [64, 15, 27, allowing complex higher entropy structures to form later on. But that effects how the arrow of time works out, leading to the Second Law of Thermodynamics, rather than its very existence; that is provided by the EBU context.

\subsection{Closed timelike lines: Chronology protection}

A longstanding problem for general relativity theory is that closed timelike lines can occur in exact solutions of the Einstein Field Equations with reasonable matter content, as shown famously in the static rotating Gödel solution [49]. This opens up the possibility of many paradoxes, such as killing your own grandparents before you were born and so creating causally untenable situations.

It has been hypothesized that a Chronology Protection Conjecture [48 would prevent this happening. Various arguments have been given in its support 79, but this remains an ad hoc condition added on as an extra requirement on solutions of the field equations, which do not by themselves give the needed protection.

The EBU automatically provides such protection, because creating closed timelike lines in this context requires the undetermined part of spacetime intruding on regions that have already been fixed. But the evolving spacetime regions can never intrude into the completed past domains and so create closed timelike lines through some spacetime event $P$, because to do so would require the fundamental world lines to intersect each other either before reaching $P$, or at $P$. Assuming plausible energy conditions, that would create a space-time singularity [49, because (being timelike eigenvectors of the Ricci tensor) they are the average flow lines of matter, and in the real universe, there is always matter or radiation present: $R_{a b} \neq 0$. The extension of time cannot be continued beyond such singularities, because they are the boundary of spacetime.

Causality: The existence of closed timelike lines ([15]:93-116) is prevented in an EBU, because if the fundamental world lines intersect, a spacetime singularity occurs [49]: the worldlines are incomplete in the future, time comes to an end there, and no "Grandfather Paradox" can occur.

Hence the EBU as outlined above automatically provides chronology protection.

\section{Overall: A more realistic view}

This paper has proposed an Evolving Block Universe (EBU) representation of spacetime which grows with time as events happen. This final section reviews how it relates to the basic features usually expected of time, and to some of the surprising features of time revealed to us by relativity theory.

The EBU model recognizes that the nature of the future is completely different from the nature of the past. The past has taken place and is fixed, and so the nature of its existence is quite different than that 
of the indeterminate future. Uncertainty exists as regards both the future and the past, but its nature is different in these two cases. The future is uncertain because it is not yet determined: it does not yet exist in a physical sense (although it is constrained in key ways by the current state of things). Thus this uncertainty has an ontological character. The past however is fixed and unchanging, because it has already happened, and the times when it happened cannot be revisited; but our knowledge about it is incomplete, and can change with time. Thus this uncertainty is epistemological in nature.

In Newtonian theory, and in ordinary quantum theory, time is the source of

1. ordering of events,

2. duration measured between events,

3. simultaneity: synchronisation of distant events,

4. direction of the flow of time,

5. transition: the fact that time flows,

6. continuity of the flow of time,

7. monotonic nature of that flow [it can't reverse or close up].

But Special Relativity and General Relativity changed that, with a surprising find [49]:

Key discovery 1: simultaneity 3. is not fundamental to time:- time flows along timelike world lines, proper time along world lines is the fundamentally preferred time parameter. It is measured by the spacetime metric, which determines duration 2.

Thus simultaneity 3 . is secondary, with no direct physical consequences. What matters are interactions between distinct entities; these take place via timelike curves and null geodesics, not on spacelike surfaces. This potentially puts a major barrier in the way of the EBU proposal where the flow of time is taken seriously, but this paper has suggested those barriers are resolved by identifying preferred timelike curves and associated spacelike surfaces in realistic models of the real universe (4.3).

Additionally, general relativity made a crucial difference to $7 .:$

Key discovery 2: - monotonicity \%. is not necessarily true in a curved spacetime, unless something prevents it (as shown by Gödel, closed timelike lines are potentially possible even for solutions of the Einstein Field Equations) [49.

The EBU model solves this key problem (\$8.2), which means ordering 1 . is also OK in them.

Unlike the Block Universe models, the General Relativity EBU models adequately represents 4., 5., and 6., which is the same in them as in Newtonian theory.

When quantum effects are significant, the future manifests all the signs of quantum weirdness, including duality, uncertainty, and entanglement. With the passage of time, after the time-irreversible process of state-vector reduction has taken place, the past emerges, with the previous quantum uncertainty replaced by the classical certainty of definite particle identities and states. The present time is where this transition largely takes place. But the process does not take place uniformly or reversibly: evidence from delayed choice experiments shows that some isolated patches of quantum indeterminacy remain, and their transition from probability to certainty only takes place later. Thus, when quantum effects are significant, the Evolving Block Universe ("EBU") of classical physics cedes way to the Crystallizing Block Universe ("CBU") [30]. On large enough scales that quantum effects are not significant, the two models become indistinguishable. 
Interesting work to be done arising of the EBU proposal, apart from testing its basic ideas, includes

1. Determining the nature of the preferred time surfaces defined in $₫ 3$ in inhomogeneous cosmologies (they are the same as the usual surfaces in spatially homogeneous models);

2. Extending the ADM analysis of $\$ 4.3$ to the case where the preferred surfaces of constant time go null and then become timelike;

3. Relating the geometry of time surfaces when spacetime is represented on different averaging scales; this is an aspect of the fitting and averaging problem for general relativity theory [22, 32];

4. Determining how the idea can sensibly relate to entanglement and EPR type experiments [1, 44];

5. Investigating the relation of quantum gravity theories to the EBU proposal.

We do not yet have a reliable theory of quantum gravity, but there are some proposals that do indeed see time at the quantum level as unfolding in a way analogous to the EBU (for example spin foam models [6]). My view would be that however they relate to time [51, 52, 13, 14, 50, 2, they must be capable of producing an EBU at the classical level, or they will fail the fundamental test of relating convincingly to the physics of ordinary everyday life. This is a correspondence principle for these theories.

Conclusion: I have reviewed the many arguments against the flow of time, in particular those based in the Wheeler-de Witt equation, and have argued that they do not carry the day: the EBU is a good model of spacetime that fits well with our daily experience as well as with general relativity and quantum theory. A key issue is how the properties of time relate to the experiences we have through the operations of our mind; I have argued ( $(6)$ that this is crucial evidence we must take into account:

A key test: The experimental evidence supporting the huge corpus of present-day neuroscience [55] decisively favors the EBU over the usual Block Universe proposal, at the classical level; therefore to be acceptable, any proposed underlying theory must pass the critical test of leading to an effective EBU at the macro level.

The physics equations we should believe are those that are compatible with this evidence; those that are not fail a basic reality test.

Acknowledgement: I thank R Goswami, C Clarkson, R Tavakol, and T Clifton for helpful comments.

\section{Appendix: R Goswami}

This Appendix by $\mathrm{R}$ Goswami develops further the relation of the proposal made here to the usual ADM formalism (see 4.3 ).

Let us consider a globally hyperbolic manifold $(\mathcal{M}, g)$, having a topological structure $\Sigma \otimes \mathbf{R}$, where $\Sigma_{t}$ denotes the family of spacelike hypersurfaces labelled by the parameter $t$. On each hypersurface of constant $t$, we can define a purely spatial metric as $h_{a b}=g_{a b}+n_{a} n_{b}$ where $n^{a}$ is the (necessarily timelike) unit normal vector of $\Sigma_{t}$ with $n^{a} n_{a}=-1$. Hence, given the foliation on $(\mathcal{M}, g)$, the spatial metric $h_{a b}$ on the spacelike hypersurface $\Sigma_{t}$ is uniquely defined. 
Let us also assume, that the Ricci tensor $R_{a b}$ on this manifold, has one timelike and three spacelike eigenvectors. These eigenvectors are unique for any physically realistic (Type $I$ ) non-zero matter field. Let the time-line for a given observer, be the integral curve of the timelike eigenvector $t^{a}$ of $R_{a b}$. This then uniquely defines the shift-vector with respect to a given foliation of a family of spacelike hypersurfaces $\Sigma_{t}$ as

$$
N^{a}=h_{b}^{a} t^{b}
$$

Furthermore, if we specify the relation between this co-ordinate time ' $t$ ' and proper time ' $\tau^{\text {' }}$ ' as $d \tau=$ $N\left(t, x^{i}\right) d t$, (where $x^{i}$ are the co-ordinates on the 3 -surface $\Sigma_{t}$ ), then by definition this gives the lapse function

$$
N\left(t, x^{i}\right)=-t^{a} n_{a}
$$

Specifically if the co-ordinate time is equal to the proper time the we must have $t^{a} n_{a}=-1$.

\section{References}

[1] Y Aharanov and D Rohrlich (2005): Quantum Paradoxes. Quantum Theory for the Perplexed (Weinheim: Wiley-VCH Verlag).

[2] Edward Anderson (2012) "Problem of Time in Quantum Gravity": arXiv:1206.2403

[3] P W Anderson (1972) "More is different" Science 177: 393-396.

[4] Peter Anninos (2001) "Computational Cosmology: From the Early Universe to the Large Scale Structure" Living Rev. Relativity 4:2 http://relativity.livingreviews.org/Articles/lrr-2001-2/].

[5] Richard Arnowitt, Stanley Deser and Charles W. Misner (1962) "The Dynamics of General Relativity". In Gravitation: An Introduction to Current Research, Ed. Louis Witten (Wiley): 227-265. Reprinted in Gen. Rel. Grav. 40: 1997 (2008).

[6] John C. Baez (1998) "Spin Foam Models" Class.Quant.Grav. 15 (1998) 1827-1858 arXiv:gr-qc/9709052v3].

[7] J B Barbour (1999) The End of Time: The Next Revolution in Phyiscs (Oxford: Oxford University Press).

[8] H.-P Breuer and F Petruccione (2006) The Theory of open quantum systems (Oxford: Clarendon Press).

[9] J.S. Briggs and J.M. Rost: "Time dependence in quantum mechanics" Eur. Phys. J. D 10, 311-318.

[10] John S. Briggs and Jan M. Rost (2001) "On the Derivation of the Time-Dependent Equation of Schroödinger" Foundations of Physics: 31:693-712.

[11] C D Broad (1923), Scientific Thought (New York: Harcourt, Brace and Co.). For Table of Contents and some chapters, see http://www.ditext.com/broad/st/st-con.html.

[12] J. N. Butterfield (2010) "The End of Time?" arXiv:gr-qc/0103055.

[13] J. Butterfield and C.J.Isham (1999) "On the Emergence of Time in Quantum Gravity" In The Arguments of Time, ed. J. Butterfield, Oxford University Press, 1999 arXiv:gr-qc/9901024v1]. 
[14] J.Butterfield and C.J.Isham (2000) "Spacetime and the Philosophical Challenge of Quantum Gravity" In Physics meets Philosophy at the Planck Scale, ed. C. Callender and N. Huggett, Cambridge University Press (2000) arXiv:gr-qc/9903072v1].

[15] S Carroll (2010) From Eternity to here: the quest for the ultimate arrow of time (New York: Dutton).

[16] P C W Davies (1974), The physics of time asymmetry. (Surrey University Press, London).

[17] P C W Davies (2012), "That Mysterious Flow". Scientific American Special Edition: A Matter of Time Vol 21: 2012), 8-13.

[18] S Dodelson (2003) Modern Cosmology (New York: Academic Press).

[19] A S Eddington (1928). The Nature of the Physical World. (London: MacMillan).

[20] G F R Ellis (1967) "The dynamics of pressure-free matter in general relativity". Journ Math Phys 8, 1171-1194.

[21] G F R Ellis (1971) "Relativistic Cosmology". In General Relativity and Cosmology, Proc Int School of Physics "Enrico Fermi" (Varenna), Course XLVII. Ed. R K Sachs (Academic Press, 1971), 104-179. Reprinted as Golden Oldie:: Gen. Rel. Grav. 41: 581 (2009).

[22] G F R Ellis (1984): "Relativistic cosmology: its nature, aims and problems".In General Relativity and Gravitation, Ed B Bertotti et al (Reidel, 215-288.

[23] G F R Ellis (2002) "Cosmology and Local Physics". New Astronomy Reviews 46: 645-658 ( gr-qc/0102017).

[24] G F R Ellis (2006) "Physics in the Real Universe: Time and Spacetime". GRG 38:1797-1824 arXiv:gr-qc/0605049.

[25] G F R Ellis (2008) "On the nature of causation in complex systems" Trans Roy Soc South Africa 63 : $69-84$.

[26] G F R Ellis (2012) "On the limits of quantum theory: contextuality and the quantum-classical cut" Annals of Physics 327 1890-1932 arXiv:1108.5261].

[27] George F R Ellis (2011) "The arrow of time, the nature of spacetime, and quantum measurement"

$\backslash$ protect \vrule width0pt\protect \href\{http://www.mth.uct.ac.za/\string ${ }^{2}$ llis/Quantum_arrowoftime_gf

[28] A R King and G F R Ellis (1973): "Tilted homogeneous cosmologies". Comm Math Phys 31, 209-242.

[29] G F R Ellis and D R Matravers: "Spatial Homogeneity and the size of the universe". In A Random Walk in Relativity and Cosmology (Raychaudhuri Festschrift). Ed N Dadhich, J K Rao, J V Narlikar, and C V Vishveshswara. (Wiley Eastern, Delhi. 1985), 92-108.

[30] G F R Ellis and T Rothman (2010): "Crystallizing block universes". International Journal of Theoretical Physics 49: 988. arXiv:0912.0808.

[31] G F R Ellis and D W Sciama: "Global and non-global problems in cosmology". In General Relativity (A Synge Festschrift), ed. L. O'Raifeartaigh (Oxford University Press, 1972), 35-59. 
[32] G F R Ellis and W R Stoeger (1987): "The Fitting Problem in Cosmology". Class Qu Grav 4, 1679-1690.

[33] George F. R. Ellis, Henk van Elst, Jeff Murugan, Jean-Philippe Uzan (2011) "On the Trace-Free Einstein Equations as a Viable Alternative to General Relativity" Class. Quantum Grav. 28: 225007 arXiv:1008.1196.

[34] G F R Ellis and R M Williams (2000) Flat and Curved Space Times. (Oxford University Press, Second edition).

[35] R Feynman (1985) QED: The Strange Theory of Light and Matter (Princeton: Princeton University Press).

[36] R P Feynman, R B Leighton and M Sands (1963) The Feynman lecturs on Physics: Mainly Mechanics, Radiation, and Heat (Reading, Mass: Addison-Wesley).

[37] R P Feynman, R B Leighton and M Sands (1964) The Feynman lecturs on Physics: The Electromagnetic Field (Reading, Mass: Addison-Wesley).

[38] R P Feynman, R B Leighton and M Sands (1965) The Feynman lecturs on Physics: Quantum Mechanics (Reading, Mass: Addison-Wesley).

[39] FQXI essay competition (2010): http://fqxi.org/community/forum/category/10

[40] FQXI meeting on time (2011): http://fqxi.org/conference/2011.

[41] R Gambini and J Pullin (2012) A First Course in Loop Quantum Gravity (Oxford: Oxford University Press).

[42] J Gemmer, M Michel and G Mahler (2004) Quantum Thermodynamics: Emergence of Thermodynamic Behaviour Within Composite Quantum Systems (Heidelberg: Springer).

[43] G W Gibbons, E P S Shellard and S J Rankin (Eds) (2003) The Future of Theoretical physics and Cosmology: Celebrating Stephen Hawking's 60th Birthday (Cambridge: Cambridge University Press).

[44] G Greenstein and A G Zajonc (2006) The Quantum Challenge: Modern Research on the Foundations of Quantum Mechanics (Sudbury, Mass: Jones and Bartlett).

[45] J Halliwell (2003) "The interpretation of quantum cosmology and the problem of time". In The Future of Theoretical phyiscs and Cosmology: Celebrating Stephen Hawking's 60th Birthday, Ed. G W Gibbons, E P S Shellard and S J Rankin (Cambridge: Cambridge University Press), 675-690.

[46] J Hartle (2003) "Theories of everything and Hawking's wave function". In The Future of Theoretical phyiscs and Cosmology: Celebrating Stephen Hawking's 60th Birthday, Ed. G W Gibbons, E P S Shellard and S J Rankin (Cambridge: Cambridge University Press), 38-49 and 615-620.

[47] S W Hawking (1984) "The quantum state of the universe" Nucl. Phys. B239, 2447.

[48] S W Hawking (1992) "The chronology protection conjecture". Phys. Rev. D46, 603-611.

[49] S W Hawking and G F R Ellis (1973): The Large Scale Structure of Space-Time. (Cambridge: Cambridge University Press). 
[50] Nick Huggett, Tiziana Vistarini, and Christian Wuthrich (2012) "Time in quantum gravity". To appear, The Blackwell Companion to the Philosophy of Time, Ed Adrian Bardon and Heather Dyke arXiv:1207.1635.

[51] Chris J. Isham (1992) "Canonical Quantum Gravity and the Problem of Time" Lectures at the NATO Summer School held in Salamanca gr-qc/9210011.

[52] C.J.Isham (1993) "Prima Facie Questions in Quantum Gravity" arXiv:gr-qc/9310031v1

[53] C J Isham (1997) Lectures on Quantum Theory: Mathematical and Structural Foundations (London: Imperial College Press).

[54] Vincent Jacques, E. Wu, Frederic Grosshans, Francois Treussart, Philippe Grangier, Alain Aspect, JeanFrancois Roch (2007). "Experimental realization of Wheeler's delayed-choice GedankenExperiment" Science 315, 5814 [arXiv:quant-ph/0610241v1].

[55] E R Kandel, J H Schwartz, and T M Jessell (2000) Principles of Neuroscience (New York: McGraw Hill).

[56] I Kanter, Y Aviad, I Reidler, E Cohen, and M Rosenbluh (2010). "An optical ultrafast random bit generator". Nature Photonics, 4: 5861.

[57] Yoon-Ho Kim, R. Yu, S.P. Kulik, Y.H. Shih, Marlan O. Scully [2000] "A Delayed Choice Quantum Eraser" Phys.Rev.Lett. 84:1-5 arXiv:quant-ph/9903047v1].

[58] Oleg Kupervasser, Hrvoje Nikoli, Vinko Zlati (2012) "The Universal Arrow of Time": arXiv:1011.4173

[59] D H Mellor, Real Time II. (Routledge, London: 1998).

[60] N.F. Mott (1931) "Time dependence in quantum mechanics" Proc. Camb. Phil. Soc. 27, 553.

[61] J V Narlikar (1996) The lighter side of gravity (Cambridge University Press).

[62] R Penrose (1989) The Emperor's New Mind (Oxford: Oxford University Press).

[63] R Penrose (2004) The Road to Reality: A complete guide to the Laws of the Universe (London: Jonathan Cape).

[64] R Penrose (2011) Cycles of Time: An Extraordinary New View of the Universe (New York: Knopf).

[65] I Percival (1991), "Schrödinger's quantum cat". Nature 351, 357.

[66] Asher Peres (1997) "Critique of the Wheeler-DeWitt equation" In On Einstein's Path ed. by A. Harvey (Springer, 1998) pp. 367-379 arXiv:gr-qc/9704061 v2].

[67] H Price (1996) Time's Arrow and Archimedes' Point (New York: Oxford University Press).

[68] M J Rees (1995) Perspectives in astrophysical cosmology (Cambridge: Cambridge University Press).

[69] C Rovelli (2008) "Forget time" FQXI essay, http://fqxi.org/community/forum/topic/237. 
[70] S Savitt, "Being and Becoming in Modern Physics", In The Stanford Encyclopedia of Philosophy (Spring 2002 Edition), Edward N. Zalta (ed.), see http://plato.stanford.edu/archives/spr2002/entries/spacetime-bebecome/.

[71] J Scalo, J Craig Wheeler and P Williams (2001), Intermittent jolts of galactic UV radiation: Mutagenetic effects In Frontiers of Life; 12th Rencontres de Blois, ed. L. M. Celnikier astro-ph/0104209.

[72] Scientific American Special Edition (2012): A Matter of Time Vol 21: 8-13.

[73] Lee Smolin (2009) "Quantization of unimodular gravity and the cosmological constant problems" Phys. Rev. D 80084003 arXiv:0904.4841v1 [hep-th].

[74] Lee Smolin (2010) "Unimodular loop quantum gravity and the problems of time": arXiv:1008.1759.

[75] Dava Sobel (1995) Longitude: The True Story of a Lone Genius Who Solved the Greatest Scientific Problem of His Time (Walker and Company).

[76] R.D. Sorkin, 'On the Role of Time in the Sum-over-histories Framework for Gravity Int." J. Theor. Phys. 33:523-534 (1994); R Sorkin Spacetime and causal sets (1991) http://www.cdms.syr.edu/ sorkin/some.papers/66.cocoyoc.pdf.

[77] Murray R. Spiegel (1967) Theory and Problems of Theoretical Mechanics (Schaum - McGraw-Hill).

[78] K S Thorne )(1995) Black Holes and Time Warps: Einstein's Outrageous Legacy (W. W. Norton and Company).

[79] M Visser (2002) "The quantum physics of chronology protection". In The Future of Theoretical Physics and Cosmology: Celebrating Stephen Hawking's 60th Birthday Ed G W Gibbons, E P S Shellard and S J Rankin (Cambridge: Cambridge University Press), 161-173 [arXiv:gr-qc/0204022v2].

[80] W. G. Unruh (1989) “A Unimodular Theory Of Canonical Quantum Gravity" Phys.Rev.D40: 1048.

[81] W. G. Unruh and R.M.Wald (1989) "Time And The Interpretation Of Canonical Quantum Gravity". Phys.Rev. D40:2598.

[82] Steven Weinberg (1989) "The cosmological constant problem" Rev. Mod. Phys. 61 1-23. 13.

[83] John Archibald Wheeler (1978), "The 'Past' and the 'Delayed-Choice Double-Slit Experiment'," pp 9-48 in A.R. Marlow, editor, Mathematical Foundations of Quantum Theory, Academic Press.

[84] J. A. Wheeler and R. P. Feynman (1945), "Interaction with the Absorber as the Mechanism of Radiation". Rev. Mod. Phys. 17, 157-181.

[85] H-D Zeh (2007) The Physical Basis of the Direction of Time (Berlin: Springer Verlag).

Author emails:

George Ellis: gfrellis@gmail.com

Rituparno Goswami: vitasta9@gmail.com

Version 2012-08-26. 